Voix et Images

voixetimages

\title{
L'extrême fixité des choses qui passent
}

\section{Michel Biron}

Volume 30, numéro 3 (90), printemps 2005

La littérature anglo-québécoise

URI : https://id.erudit.org/iderudit/011863ar

DOI : https://doi.org/10.7202/011863ar

Aller au sommaire du numéro

\section{Éditeur(s)}

Université du Québec à Montréal

ISSN

0318-9201 (imprimé)

1705-933X (numérique)

Découvrir la revue

Citer ce compte rendu

Biron, M. (2005). Compte rendu de [L'extrême fixité des choses qui passent].

Voix et Images, 30(3), 153-159. https://doi.org/10.7202/011863ar d'utilisation que vous pouvez consulter en ligne.

https://apropos.erudit.org/fr/usagers/politique-dutilisation/ 


\author{
R O M A N \\ L'extrême fixité des choses qui passent \\ $+++$ \\ MICHEL BIRON \\ Université McGill
}

Les quatre romanciers dont il sera question ci-dessous sont des hommes nés il y a soixante ans ou plus, tous publiés chez le même éditeur (Boréal) et qui raconte une histoire dans laquelle le personnage principal est un écrivain ou un éditeur littéraire. Est-ce assez pour en tirer un portrait de groupe, celui d'une génération ou d'une maison d'édition? Les quatre romans ont en commun l'élégance du style, des allusions nombreuses à la littérature et au milieu littéraire, une intrigue romanesque minimale ou secondaire, des héros assez quelconques qui habitent Montréal et qui vivent à l'époque actuelle. Or, de cette époque, justement, aucun de ces héros ne semble se satisfaire. Chacun entre en lutte avec ce qu'il a été, avec ce qu'il est devenu, le passé et le présent se mêlant dans une difficile appréhension de soi. Avec tantôt des accès de pessimisme à la Émile Cioran, tantôt des instants de grâce où le temps paraît s'alléger indéfiniment.

Le siècle de Jeanne ${ }^{1}$ n'est que le cinquième roman d'Yvon Rivard, dont la carrière a commencé en 1976 avec Mort et naissance de Christophe Ulric, un roman audacieux acclamé à l'époque par Hubert Aquin et par Pierre Vadeboncœur. Même s'il a reçu par la suite quelques prix prestigieux, dont le Prix du Gouverneur général pour Les silences du corbeau (1986) et le Grand Prix du livre de Montréal pour Le milieu du jour (1995), Rivard demeure un auteur rare, à qui il arrive même de se montrer cynique à l'endroit de la machine littéraire, comme lorsqu'il fait dire au narrateur de son dernier roman que "plus on diffuse les œuvres, moins il y a d'art» (127). Ce n'est pas ainsi, sans doute, que l'on envisage la littérature dans les organismes gouvernementaux chargés d'en faire la promotion. Est-ce pour cela qu'il n'est guère connu en dehors d'un cercle de fidèles lecteurs? Il y a peut-être une autre raison, plus profonde, qui tient au fait que son œuvre ne s'identifie pas tellement à la

$$
+++
$$

1 Yvon Rivard, Le siècle de Jeanne, Montréal, Boréal, 2005, 401 p. 
littérature québécoise, sauf dans le cas d'Hector de Saint-Denys Garneau à qui l'auteur a consacré des essais remarquables ${ }^{2}$. Rivard ne parvient à être lui-même qu'à une certaine distance et à une certaine hauteur, parmi des poètes comme Rainer Maria Rilke ou Freidrich Hölderlin, ou des romanciers comme Peter Handke et surtout Virginia Woolf, plusieurs fois citée dans Le siècle de Jeanne. Ses premiers romans, hantés par l'idéalisme et le romantisme allemand, étaient peuplés de femmes irréelles, situés dans des pays sans frontières et portés par d'ambitieuses questions philosophiques. Puis, le romancier a commencé à toucher terre ( «Je suis un romantique qui se soigne», dira-t-il avec humour dans un de ses essais ${ }^{3}$ ), d'abord très loin de chez lui, à Pondichéry où se déroule Les silences du corbeau, puis à Montréal, dans Le milieu du jour. Mais la passion romantique n'est jamais disparue, comme on le voit dans Le siècle de Jeanne, qui est son roman à la fois le plus maîtrisé et le plus émouvant.

Au cœur du Siècle de Jeanne, on trouve la même situation, le même triangle amoureux, les mêmes personnages torturés et malheureux que dans les deux romans précédents. Mais le temps a passé et il y a déjà six ans qu'Alexandre habite seul. Entre les scénarios qu'il écrit pour gagner sa vie, il continue d'aimer Clara, qui l'a quitté, et rêve plus que jamais de réparer le désastre de son autre vie, celle qu'il menait avec sa femme Françoise et sa fille Alice. Impossible de tourner la page, le passé refuse de se laisser oublier. C'est d'ailleurs l'un des grands thèmes de ce roman qui est une sorte de méditation sur le temps. Alexandre est obsédé par ce que Virginia Woolf appelait magnifiquement "l'extrême fixité des choses qui passent» (87 et 147). D'où la passion que lui inspire l'enfance avec ses petits gestes, ses jeux apparemment insignifiants et sans cesse répétés. C'est le temps qu'incarne Jeanne, la fille d'Alice. Elle est née avec ce siècle, ou presque, et elle représente pour Alexandre l'innocence retrouvée, celle par qui il rachète, peut-être, les fautes anciennes. Il aime Jeanne comme il n'a pas réussi à aimer les autres femmes de sa vie, ni Françoise ni Clara et encore moins sa fille Alice, brutalement abandonnée lorsque Alexandre est tombé amoureux de Clara. Faute capitale, comme il le dira en citant Gœthe, celle d'un père qui a préféré ses propres passions (écrire, aimer) aux désirs et aux peurs de son enfant. C'est là, dans ces pages bouleversantes de vérité, que le romancier donne sa pleine mesure, avec une clarté d'expression admirable et une lucidité qui semblent s'accroître au fur et à mesure qu'on entre dans le vif de la blessure, de la faute.

À travers les déchirements amoureux d'Alexandre, Le siècle de Jeanne aborde aussi d'autres thèmes qui donnent parfois au roman l'allure d'un essai, mais d'un essai qui se nourrit de la fiction et qui s'interroge sur le monde à travers la littérature, l'art ou le cinéma. Il est notamment beaucoup question d'Amérique, en particulier dans son opposition à l'Ancien Monde ou dans ses rapports troubles avec la beauté de ses propres paysages comme avec la laideur de ses villes. Alexandre se demande pourquoi c'est à Paris que le Québécois se découvre tel qu'il est, c'est-

$$
++
$$

2 Id., "Qui a tué Saint-Denys Garneau? ", Liberté, n 139, 1982, p. 73-85. Voir aussi «L'héritage de la pauvreté», Littératures, no 17, 1998, p. 205-219. 3 Id., Le bout cassé de tous les chemins, Montréal, Boréal, coll. «Papiers collés», 1993, p. 65. 
à-dire comme un habitant du Nouveau Monde. À Montréal, au contraire, le Québécois doit sans cesse lutter contre sa mélancolie, qui menace de verser dans le vide ou la folie alors que dans l'Ancien Monde, là où la beauté a un sens, la mélancolie est aussi une forme d'émerveillement, de présence à soi et aux autres:

\begin{abstract}
Pourquoi cette menace constante de la détresse au sein même de la joie, comme si la joie ici longeait toujours quelque précipice? Était-ce le prix à payer pour vivre dans l'éternité mobile de l'instant que cette absence de balises qui nous expose au chaos et au découragement, comme si vivre ici n'était pas un métier mais une aventure dans laquelle le souci sédentaire de beauté et d'harmonie n'avait pas sa place? (287)
\end{abstract}

Le chaos qui fascine et désespère tant Alexandre, c'est l'Amérique, c'est Montréal, $c^{\prime}$ est l'appartement en désordre de Clara et c'est surtout Clara elle-même qui n'arrête pas de vouloir se tuer. Pourtant, ce roman immensément mélancolique laisse à la fin une impression d'étonnante sérénité, mélange d'équilibre et de passion. À cause de Jeanne d'abord qui illumine la vie d'Alexandre, mais aussi par la magie même de l'écriture qui arrive à arracher le personnage au désordre temporel, à donner à certains moments une plénitude particulière, une intensité susceptible de se répéter, de s'engendrer à nouveau. Ainsi, la mélancolie peinte par Yvon Rivard est infiniment variée dans sa continuité. Elle est tout sauf grise, comme une promesse de création qui produit un sentiment de joie en même temps qu'elle s'ouvre à la tragédie de l'existence. C'est la vie même qui se donne à lire, avec ses éblouissements furtifs et cette conscience allégée qui traque l'éternité dans ce qui est le plus fragile, dans cette "nouvelle culture de l'instant» (288) qui, loin d'abolir le passé, l'élargit au point de rapprocher, dans un seul et même geste, le commencement et la fin du monde.

À l'inverse d'Yvon Rivard, Gilles Archambault est un écrivain prolifique, qui compte déjà une vingtaine de romans ou de recueils de nouvelles, auxquels s'ajoutent quantité de chroniques. Mais chacune des fictions d'Archambault, plus obstinément que chez Rivard, raconte toujours à peu près la même histoire. Le héros est doucement malheureux, désespéré mais pas trop, juste assez pour savourer «les plaisirs de la mélancolie", selon le titre d'un des essais de Gilles Archambault ${ }^{4}$. Ces plaisirs s'amenuisent-ils avec l'âge? Peut-être, mais les héros de ses romans ont l'habitude d'être vieux, pour ainsi dire. Le thème de la vieillesse a toujours été présent dans son œuvre, dès son premier roman Une suprême discrétion, écrit en pleine Révolution tranquille ${ }^{5}$. À l'époque, les romans d'Archambault se distinguaient

$$
++
$$

4 Gilles Archambault, Les plaisirs de la mélancolie. Petites proses presque noires, Montréal, Quinze, 1980.

5 Id., Une suprême discrétion, Montréal, Cercle du livre de France, 1963. 
nettement des romans dits «modernes» de la littérature québécoise. Ils ne collaient pas à l'image du jeune écrivain qu'on se faisait à Parti pris ou à Liberté. On voit bien, par là, à quel point Archambault ne s'est jamais soucié d'être de son temps, au risque d'être marginalisé par l'histoire littéraire, comme ces écrivains un peu oubliés auxquels il est resté fidèle et qu'il cite tout au long de son œuvre. De l'autre côté du pont ${ }^{6}$, son plus récent roman, en évoque plusieurs: Paul Morand, Alexandre Vialatte, José Cabanis, Marcel Arland, Dino Buzzati, Georges Perros et Philippe Jaccottet.

Ce roman se déroule le jour du soixante-quinzième anniversaire de Louis Audry. Au matin, il se prépare à recevoir quelques visiteurs dans sa maison trop grande du Plateau Mont-Royal. Il n'y en aura pas beaucoup, se dit-il, anticipant une journée particulièrement déprimante. Par hasard, c'est aussi ce jour-là que doit venir son ami Raymond Boujut avec le brouillon de la préface qu'il rédige pour l'édition des "EFuvres complètes» de Louis Audry. Car ce dernier est écrivain, en plus d'avoir été un éditeur important de Montréal. Son ancienne maison d'édition veut lui rendre hommage en rééditant en un seul volume ses cinq romans. L'initiative ne le touche qu'à peine, mais il accepte de jouer le jeu. Du reste, la préface en question remplit une autre fonction dans le roman. Elle permet à Archambault de brosser un autoportrait ironique, et de retoucher l'image habituelle d'écrivain mélancolique que la critique lui a accolée et qu'il a lui-même cultivée à l'occasion: "Ses romans, écrit le préfacier de Louis Audry, donnent de la vie une image qui est loin d'être rose. On chercherait en vain dans ses romans des intrigues faciles ou des personnages vainqueurs. C'est le monde de la défaite. S'il y a une force à laquelle il a cru, c'est celle des faibles. Continuer malgré les difficultés inhérentes à la condition humaine, telle est la règle que suivent ses personnages.» (109) Le mot clef est ici le verbe "continuer", qui résume bien la destinée des personnages d'Archambault. C'est aussi ce qui caractérise Louis Audry, qui se donne des airs de nostalgique pour mieux se tourner vers les jeunes et les enfants. «J'ai toujours été du côté des fils» (112), affirme-t-il résolument. Il se laisse charmer par la beauté de Pascale, une étudiante venue acheter quelques livres de sa collection. Il attend avec impatience la venue de ses enfants, qui passeront lui souhaiter un joyeux anniversaire. Et puis il y a Monique, son ancienne secrétaire et maîtresse, chez qui il trouvera finalement refuge au terme de cette journée qui le bombarde de souvenirs. La vie continue ainsi, comme si de rien n'était.

Mais comment dire la monotonie de l'existence dans une forme qui ne soit pas elle-même ennuyeuse? C'est tout le défi de ce roman, et peut-être même de l'ensemble des ouvrages de Gilles Archambault. L'écriture ne vise pas la grandeur, mais l'exactitude du trait, ce qu'elle ne réussit pas toujours. Les phrases brèves s'enchaînent un peu mécaniquement les unes aux autres, quelques scènes paraissent inutilement longues. Mais la franchise du personnage de Louis Audry finit par produire un effet de séduction. On se prend à aimer cette courageuse morosité qui lui est si naturelle et qui semble moins un motif de dépit qu'une façon de se

$$
++
$$

6 Id., De l'autre côté du pont, Montréal, Boréal, 2005, 196 p. 
remettre à sa place, de continuer à vivre malgré tout. Le reste, pour lui, c'est de la littérature.

Personne ne s'attendait à voir le critique Gilles Marcotte se lancer dans le genre du roman policier. C'est pourtant ce qui se produit avec Le manuscrit Phaneuf ${ }^{7}$, qui possède tous les ingrédients d'un polar sérieux: il y a un mort, bientôt deux, un détective, une enquête et même un certain suspens. Mais Le manuscrit Phaneuf ne ressemble pas pour autant à un vrai polar. Il joue à être un polar. Marcotte en cite d'ailleurs plusieurs, d'Agatha Christie à Henning Mankell, sans chercher à rivaliser avec eux. Tout l'arsenal de l'intrigue policière est à prendre au deuxième degré, hissé à l'étage de l'ironie, livré aux jeux de miroir et aux énigmes qui ne sont pas seulement policières. Le héros, Julien Brossard, est un éditeur connu dans le milieu montréalais. Connu, vraiment? C'est ce qu'on croit au début, mais le personnage devient de plus en plus insaisissable au fur et à mesure qu'on avance dans la lecture. Par exemple, tous les cinq ou six mois, il disparaît de la circulation sans explication. Ou encore, autre manière de fuir son époque, il se réfugie dans l'œuvre d'Honoré de Balzac, dont il possède toujours un livre dans le tiroir supérieur de son bureau. Pourquoi Balzac précisément? C'est «parce qu'il est l'inactuel même, une sorte de monstre préhistorique» (57).

Ce privilège accordé à l'inactuel donne le ton à l'ensemble du roman. L'autre personnage principal, Arcade Phaneuf, est, lui aussi, une sorte de "monstre préhistorique», mais à saveur canadienne-française. Le nom même fait sourire, comme si le mot «neuf» contenu dans le patronyme était habillé à l'ancienne, renvoyé à une époque où les enfants s'appelaient Arcade. C'est lui la première victime, noyé de façon mystérieuse au bout du quai devant son chalet des Laurentides. À la fin des années 1950, il avait écrit un roman scandaleux, Les pharisiens, puis il était devenu fonctionnaire, journaliste et même sénateur. Juste avant de mourir, il était venu soumettre un manuscrit à Julien Brossard. Or, celui-ci ne retrouve plus le «manuscrit Phaneuf» et, confus, se met à le chercher partout. De fil en aiguille, il apprend que cet homme apparemment respectable était en vérité, selon ses proches, tantôt un «salaud», tantôt un "désespéré». Au milieu du roman, on découvre qu'à peu près tout le monde avait de solides raisons de vouloir le tuer, mais qu'il a très bien pu se suicider.

Le tableau s'embrouille donc au fur à mesure qu'il s'élabore. Au lieu d'éclairer ce que cachent les différents protagonistes, le roman leur découvre des zones d'ombre et s'amuse à les couvrir de gris. Même processus de brouillage pour l'autre mort, l'éditeur Alfred Vleminckx ${ }^{8}$ qui est un collègue de Julien Brossard; ou pour le frère d'Arcade, le chanoine Phaneuf, grand lecteur de Georges Bernanos (auteur, lui

$$
+++
$$

7 Gilles Marcotte, Le manuscrit Phaneuf, Montréal, Boréal, 2005, 219 p. Marcel (143), mais par erreur sans doute.

8 Lequel se prénomme tout à coup 
aussi, d'un étonnant roman policier ${ }^{9}$ ) et sorte de résistant plutôt mal en point d'un clergé devenu une espèce rare; ou encore pour l'inspecteur Grandmaison, qui se prend pour Maigret et finit par lâcher la profession afin de se convertir finalement à la littérature; et il faudrait parler des personnages féminins, moins «littéraires» que leurs homologues masculins, et peut-être en cela plus profondément énigmatiques qu'eux. On ne saura pas trop qui est Simone, la maîtresse de Julien Brossard, ni Audrey Phaneuf, la femme d'Arcade, ni la pauvre Joanna, à moitié folle et droguée. Ce sont des personnages attirants, qu'on ne fera toutefois qu'effleurer, comme des figures d'un monde à la fois ordinaire et secret où chacun n'est pas tout à fait sûr de savoir d'où il vient ni où il va. Il faudra se contenter de petits détails, livrés souvent en apartés, le narrateur ne se préoccupant pas de faire entrer le lecteur dans l'intimité des personnages. Mais ce sont ces détails, ces indices qui permettent de pressentir la fragilité de chacun d'entre eux, en particulier de ceux qui, durant toute leur vie, ont passé pour des hommes efficaces et sûrs d'eux. D'ailleurs, le roman vaut beaucoup plus par l'ambiguïté et les contradictions de ces portraits que par les événements propres à l'intrigue policière. On lira ainsi les très belles scènes, intercalées tout au long du roman, qui tentent de reconstituer le tableau central, celui qui montre Arcade Phaneuf au bout du quai, au moment de l'événement tragique. Avec l'élégance et la précision qui caractérisent son écriture, Gilles Marcotte ne se satisfait pas de faire du roman policier une machine à produire la vérité: il casse l'image trop commode, trop simple que nous aimons avoir des autres ou de nous-même.

La brûlerie ${ }^{10}$, roman posthume d'Émile Ollivier, mort en 2002, est le nom d'un café situé sur le chemin de la Côte-des-Neiges, tout près de l'Université de Montréal. Rien à voir ou presque avec les lieux légendaires qui ont marqué ce quartier, les Bouvillon et autres Café Campus. Ici, l'odeur du café remplace celle du houblon. Il y a des étudiants, bien sûr, mais ce ne sont pas eux que l'on voit. La brûlerie est le refuge d'un groupe d'Haïtiens vieillissants qui discutent du présent et surtout du passé. Le chemin de la Côte-des-Neiges leur appartient, comme la rue Fabre appartient aux personnages de Tremblay. Mais l'habitation des lieux n'a pourtant pas le même sens dans ce roman de l'exil. Elle tient de l'étonnement perpétuel et suscite mille interrogations qui sont de nature plus sociologique que romanesque (Émile Olliver, rappelons-le, était sociologue de métier). Les personnages ne cessent d'éprouver aussi bien la résistance de la société québécoise à leur endroit que leur propre résistance face à leur société d'accueil. L'intention est nette: «[U]n vrai travail d'écriture sur Montréal devrait commencer par mettre en scène la parole nomade, la parole migrante, celle d'entre-deux, celle de nulle part, celle d'ailleurs ou d'à côté, celle de pas tout à fait d'ici, pas tout à fait ailleurs.» (55-56) Montréal n'est plus pour ces personnages venus d'ailleurs la ville aux deux solitudes de Hugh

$$
++
$$

9 Georges Bernanos, Un crime, Paris, Plon, 1935.

10 Émile Ollivier, La brûlerie, Montréal, Boréal, 2005, 246 p. 
MacLennan, mais plutôt la "ville aux quatre solitudes - celle d'être francophone, anglophone, immigrant et noir» (56). Ils s'inventent un «Ministère de la Parole» où ils causent de la patrie natale, de l'insupportable hiver ou des femmes québécoises qu'ils jugent beaucoup trop susceptibles. Ils ont des noms de dieux, comme Dionysos d'Acapulco, ou de poètes comme Virgile ou Homère. Mais ce sont des dieux déchus, des poètes brûlés par la vie et pour qui les thèmes si à la mode de l'errance et de l'exil ne sont pas des métaphores, mais une réalité qui structure toute leur vie.

Ce roman montréalais n'a toutefois pas la cohérence et la limpidité des autres textes à caractère autobiographique d'Émile Ollivier, comme le très beau récit Mille eaux ${ }^{11}$, qui évoque l'enfance haïtienne de l'auteur. L'écriture est tiède et elle a parfois quelque chose de convenu, de lourdement idéologique, comme lorsque le narrateur dénonce l'arrivée des fast-foods qui viennent enlaidir le chemin de la Côte-des-Neiges. Les passages les plus émouvants sont ceux où le narrateur oublie le langage de la sociologie pour brosser le portrait de ses amis et raconter leur vie étrange. On lira en particulier les pages splendides consacrées au personnage de Virgile, un éternel migrant qui a vécu dans six villes différentes avant de se retrouver à Montréal, où il ne s'est jamais senti chez lui. Entre cette ville et lui, il y a «incompatibilité existentielle» (122), explique le narrateur. Mais l'incompatibilité ne se limite pas à la ville. L'homme qui aimait bien raconter des énigmes à ses amis du «Ministère de la Parole» est rongé par quelque désespoir secret qui le pousse au suicide.

«À La Brûlerie, on pouvait observer du temps à l'état pur, à l'état naturel, comme on le dit de l'or, du temps subi ou assumé en attendant la mort.» (122) Même s'il s'agit d'un roman sur l'exil et l'errance, La brûlerie raconte avant tout une façon de s'enraciner dans le temps. C'est d'ailleurs là une obsession commune aux quatre romans évoqués dans cette chronique, et qui explique sans doute que la durée proprement romanesque y soit pervertie au profit de tableaux poétiques ou méditatifs qui figent le temps. Tous évoquent longuement le passé, mais non pas parce qu'il est perdu et qu'ils croient pouvoir le retrouver, non plus pour montrer ce qui sépare la gloire d'hier du triste aujourd'hui. Le passé est perdu, oui, comme le pays natal des personnages d'Émile Ollivier ou comme l'amour de Clara qu'Alexandre espérait faire renaître. Il y a, dans tous ces romans, un véritable désespoir qui ne plaira certainement pas aux adeptes de la littérature optimiste. Mais ce désespoir, par un paradoxe propre à la création, est peut-être ce qui permet de retrouver une sorte d'innocence dans l'écriture et une manière de se mettre à l'abri des modes et de l'actualité. Dans ces romans si peu romanesques, si pauvres en action proprement dite, l'écriture tend à s'arrêter devant le désordre des vies. Elle ne cherche pas à en épouser le cours, mais en propose au contraire une saisie verticale, un arrêt sur image, comme pour mieux sentir «l'extrême fixité des choses qui passent».

$$
++
$$

11 Émile Ollivier, Mille eaux, Paris, Éditions Gallimard, coll. «Haute enfance», 1999. 